> Dans la maladie de Fabry, l'accumulation de globotriaosylcéramide et du galabiosylcéramide dans les tissus peut être mis en évidence par différentes méthodes de coloration associées à la microscopie optique ou électronique. L'imagerie par spectrométrie de masse a été appliquée à l'analyse de ces produits accumulés dans les tissus au cours de la maladie de Fabry. Les avantages et les inconvénients de cette technique sont discutés. <

\section{Apport de l'imagerie par spectrométrie de masse pour l'analyse directe du globotriaosylcéramide et du galabiosylcéramide tissulaires}

\author{
Sandrine Roy, David Touboul, Alain Brunelle, \\ Dominique P. Germain, Olivier Laprévote, Pierre Chaminade
}

Différentes approches peuvent être mises en œuvre pour réaliser des images sur coupes tissulaires. II s'agit le plus souvent de techniques de coloration ou de fluorescence associées à la microscopie optique ou électronique. Récemment, le développement de certaines techniques de spectrométrie de masse, comme celles du MALDI-TOF (matrix assisted laser desorption ionisation time-of-flight) ou du TOF-SIMS [1-4] (time-of-flight secondary ion mass spectrometry), a permis d'élargir le panel des approches envisageables. Après un bref rappel de son principe, l'apport du TOF-SIMS sera abordé à travers l'analyse de coupes de biopsies cutanées et rénales issues de patients hémizygotes affectés par la maladie de Fabry.

Le TOF-SIMS est une technique d'analyse physico-chimique de surface de très grande sensibilité. Elle est fondée sur la détection des ions secondaires produits sous l'effet d'un bombardement d'ions primaires incidents. L'impact d'un ion possédant une énergie de quelques kilo électron-volts produit l'émission secondaire de particules de différentes natures: des photons, des électrons, des particules neutres, et des ions secondaires caractéristiques de l'échantillon. Il s'agit d'une technique d'ionisation douce, permettant ainsi d'accéder à la masse moléculaire des ions formés.

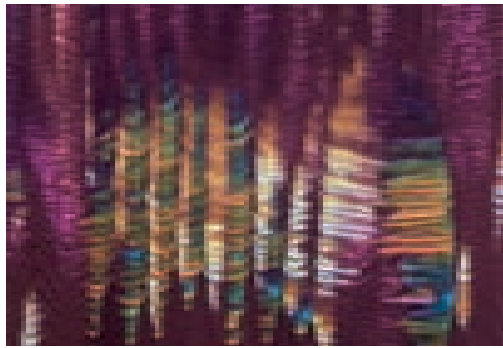

S. Roy, P. Chaminade : Groupe de Chimie Analytique du Sud de Paris, EA 3343, Faculté de Pharmacie de Paris XI, 5 , rue Jean-Baptiste Clément, 92296 Châtenay-Malabry Cedex, France.

D. Touboul, A. Brunelle, 0 . Laprévote : Laboratoire de Spectrométrie de Masse, Institut de Chimie des Substances Naturelles (ICSN-CNRS), avenue de la Terrasse, 91198 Gif-surYvette Cedex, France.

D.P. Germain : Groupe de Chimie Analytique du Sud de Paris, EA 3343, Faculté de Pharmacie de Paris XI, 5 , rue Jean-Baptiste Clément, 92296 ChâtenayMalabry Cedex, France et Unité Fonctionnelle de Génétique Clinique, Hôpital Européen Georges Pompidou, 20, rue Leblanc, 75015 Paris, France. Alain.Brunelle@icsn.cnrs-gif.fr sandrine.roy@cep.u-psud.fr
Le principe de la constitution d'une image est simple: la surface de l'image est tout d'abord déterminée. Il s'agit le plus souvent d'une surface carrée divisée en autant de lignes que de colonnes. Le nombre de lignes et de colonnes dépend de la résolution spatiale recherchée et du temps d'acquisition souhaité. Pour le TOF-SIMS, la focalisation des ions primaires permet d'accéder facilement à des résolutions spatiales de l'ordre de $1 \mu \mathrm{m}^{2}$. Des spectres de masse sont alors enregistrés à l'intérieur de chaque pixel (carré élémentaire) ainsi défini.
Un logiciel de traitement permet ensuite d'extraire, pour chacun des spectres, l'intensité des signaux correspondant à des ions de masses prédéfinies et de créer ainsi des cartes de densité ionique. Cette technique permet de réaliser, en une seule analyse, la cartographie de plusieurs biomolécules à la surface d'une biopsie tissulaire. Dans le cas de la maladie de Fabry, la mutation génétique portant sur le gène GLA codant pour l' $\alpha$-galactosidase A, deux glycosphingolipides neutres s'accumulent par 
défaut de métabolisme: le globotriaosylcéramide $\left(\mathrm{Gb}_{3}\right)$ et le digalactosylcéramide $\left(\mathrm{Ga}_{2}\right)$. L'accumulation tissulaire de ces deux lipides à la surface de coupes de biopsies cutanées et rénales a été étudiée par TOF-SIMS.

Lors de l'analyse de biopsies cutanées provenant de patients hémizygotes affectés par la maladie de Fabry avant thérapie enzymatique substitutive, nous avons observé des zones d'accumulation diffuses dans le derme et l'hypoderme. Ces zones de concentrations sont superposables pour le $\mathrm{Gb}_{3}$ et le $\mathrm{Ga}_{2}$. La spécificité de la technique a été vérifiée en procédant à l'étude de biopsies cutanées issues de volontaires sains: aucune zone d'accumulation n'ayant été retrouvée, ceci tend à confirmer que les signaux précédemment enregistrés traduisent bien la présence des glycosphingolipides non dégradés. Cependant, des études en spectrométrie de masse tandem, qui permettraient alors d'obtenir des renseignements structuraux plus précis que ceux apportés par la seule masse moléculaire, fourniraient d'avantage d'éléments de preuve.

De la même façon que pour les biopsies cutanées, l'analyse d'une biopsie rénale a permis de retrouver des zones d'accumulation superposables pour le $\mathrm{Gb}_{3}$ et le $\mathrm{Ga}_{2}$ (Figure 1). Concernant le spectre de masse moyen correspondant à la surface analysée, le rapport signalsur-bruit est suffisamment intense (supérieur à 3) pour permettre d'identifier les différentes structures lipidiques des glycosphingolipides accumulés.

$\varepsilon$ n conclusion, cette technique présente donc de nombreux avantages. Le premier concerne la rapidité avec laquelle les images peuvent être obtenues (environ 30 minutes pour les images de biopsies rénales). Le second avantage porte sur la résolution spatiale obtenue: un carré élémentaire présentant une surface de l'ordre $d u \mu m^{2}$, l'échelle de la cellule est alors atteinte. Surtout, l'étude simultanée de plusieurs molécules permet de rechercher l'existence de co-localisations ou de localisations complémentaires. Ce type d'études a récemment été réalisé sur des coupes tissulaires d'un modèle animal de myopathie de

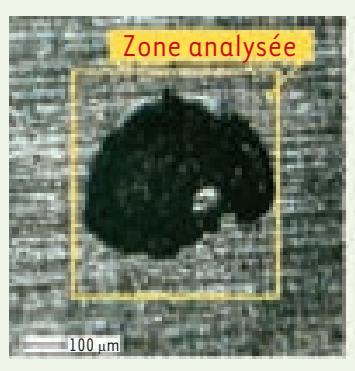

Champ de vision $500,0 \times 500,0 \mu \mathrm{m}^{2}$

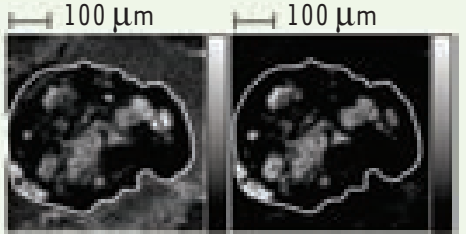

$\mathrm{Gb}_{3}$

$\mathrm{Ga}_{2}$

Figure 1. Image optique de la zone de biopsie rénale analysée et imagerie par spectrométrie de masse TOF-SIMS des zones d'accumulation des classes lipidiques du globotriaosylcéramide $\left(G_{3}\right)$ et du galabiosylcéramide $\left(G_{2}\right)_{2}$. Biopsie rénale d'un patient hémizygote affecté par la maladie de Fabry avant thérapie enzymatique substitutive. Zone de $500 \times 500 \mathrm{\mu m}^{2}$, ion primaire $=\mathrm{Bi}_{3}{ }^{+}$, dose de $6,5 \times 10^{11}$ ions $/ \mathrm{cm}^{2}$, mode d'ionisation positif, résolution spatiale: 1 pixel $=4 \mu \mathrm{m}$.
Duchenne, permettant aux auteurs de montrer que les zones de régénération tissulaire correspondaient aussi aux zones riches en anti-oxydants, comme la vitamine $\varepsilon$ [5]. Sur le même modèle, des études portant sur la colocalisation des zones de dépôts de glycosphingolipides et des marqueurs de la fibrose rénale pourraient être intéressantes dans le cas de la maladie de Fabry. Deux inconvénients peuvent toutefois être évoqués. II s'agit tout d'abord du coût de l'appareillage, qui limite son accessibilité. De plus, l'aspect quantitatif, particulièrement important dans le cas d'une maladie de surcharge et pour le suivi de l'efficacité des traitements enzymatique substitutifs, reste difficile à mettre en œuvre puisqu'il nécessiterait l'introduction d'un étalon interne au niveau de la surface de l'échantillon. $\diamond$

\section{SUMMARY}

Imaging mass spectrometry and direct analysis of globotriaosylceramide and galabiosylceramide in tissue

In Fabry disease, accumulation of globotrianosylceramide and galabiosylceramide are observed by coloration methods associated to optic or electron microscopy. But a new technology, imaging mass spectrometry, may be applied to the analysis of these metabolites in tissue of hemizygous patients with Fabry disease. Advantages and disavantadges of this technic are discussed. $\diamond$

\section{RÉFÉRENCES}

1. Stoeckli MP, Chaurand P, Hallahan DE, et al. Imaging mass spectrometry: a new technology for the analysis of protein in mammalian tissues. Nat Med $2001 ; 7: 493-6$

2. Todd PJ, Schaaff G, Chaurand P, et al. Organic ion imaging of biological tissue with secondary ion mass spectrometry and matrix-assisted laser desorption/ionization. J Mass Spectrom 2001 ; 36 : 355-69.

3. Touboul D, Halgand F, Brunelle A, et al. Tissue molecular ion imaging by gold cluster ion bombardment. Anal Chem 2004 ; 76 : 1550-9.

4. Brunelle A, Touboul D, Laprévote 0 . Biological tissue imaging with timeof-flight secondary ion mass spectrometry and cluster ion sources. J Mass Spectrom $2005 ; 40$ : 985-99.

5. Touboul D, Brunelle A, Halgand F, et al. Lipid imaging by gold cluster time-of-flight. Secondary ion mass spectrometry : application to Duchenne muscular dystrophy. J Lipid Res 2005 ; 46 : 1388-95.

\section{TIRÉS À PART}

A. Brunelle et S. Roy

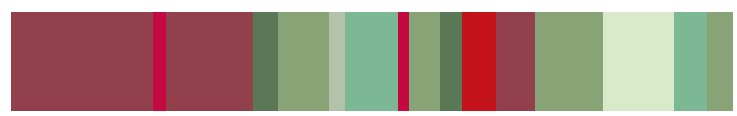

